\title{
Estados de Pseudo-Cushing
}

\begin{abstract}
RESUMO
Síndromes de pseudo-Cushing são um grupo heterogêneo de doenças, incluindo alcoolismo, anorexia nervosa, obesidade visceral e depressão, que compartilham muitas das características clínicas e bioquímicas da síndrome de Cushing. Os mecanismos responsáveis para a gênese da síndrome de pseudo-Cushing são fracamente compreendidos. Tem sido sugerido que o hipercortisolismo da síndrome de pseudo-Cushing pode ser resultante do aumento da secreção do hormônio liberador de corticotrofina ( $\mathrm{CRH}$ ) hipotalâmico no contexto de um eixo hipotálamohipofisário-adrenal que, de outra maneira, está normalmente constituído. A sobreposição substancial entre as características clínicas e laboratoriais entre muitos pacientes com síndrome de Cushing e aqueles com síndrome de pseudo-Cushing pode tornar o diagnóstico diferencial difícil. Distinguir entre síndrome de pseudo-Cushing e síndrome de Cushing verdadeira é crítico para se prevenir o tratamento desnecessário e potencialmente prejudicial de tais pacientes. Esta breve revisão sumariza os principais eventos patofisiológicos das síndromes de pseudo-Cushing e fornece uma estratégia útil para o seu diagnóstico diferencial. (Arq Bras Endocrinol Metab 2007;51/8:1303-1313)
\end{abstract}

Descritores: Síndrome de Cushing; Pseudo-Cushing; Hipercortisolismo; ACTH

\begin{abstract}
Pseudo-Cushing States.

Pseudo-Cushing syndromes are a heterogeneous group of disorders, including alcoholism, anorexia nervosa, visceral obesity, and depression, which share many of the clinical and biochemical features of Cushing's syndrome. The mechanisms responsible for the genesis of pseudo-Cushing's syndrome are poorly understood. It has been suggested that hypercortisolism of pseudo-Cushing syndrome may be the result of increased hypothalamic corticotrophin-releasing hormone (CRH) secretion in the context of a hypothalamic-pituitary-adrenal axis that is otherwise normally constituted. The substantial overlap in clinical and biochemical features among several patients with Cushing syndrome and those with pseudoCushing syndromes can make the differential diagnosis difficult. Distinguishing between pseudo-Cushing's syndrome and true Cushing's syndrome is critical for preventing the unnecessary and potentially harmful treatment of such patients. This brief review summarizes the main pathophysiological events of pseudo-Cushing syndromes and provides a useful strategy for differential diagnosis. (Arq Bras Endocrinol Metab 2007;51/8:1303-1313)
\end{abstract}

Keywords: Cushing syndrome; Pseudo-Cushing; Hypercortisolism; ACTH

\section{revisão}

\author{
DANIELLA J.P.C. ROMANHOLI \\ LUIZ ROBERTO SALGADO
}

\author{
Unidade de \\ Neuroendocrinologia da \\ Disciplina de Endocrinologia \\ do Hospital das Clínicas, \\ Universidade de São Paulo - \\ HCFMUSP, São Paulo, SP.
}

Recebido em 09/08/07

Aceito em 14/08/07 


\section{DEFINIÇÃO}

$\mathrm{E}$ STAdos de PSEUdo-Cushing (PC) podem ser definidos pela presença de um fenótipo clínico similar ao da Síndrome de Cushing (SC) verdadeira associada a alguma evidência de hipercortisolismo (1).

\section{ETIOLOGIA}

Diversas situações podem induzir ao estado de pseudo-Cushing, sendo a depressão maior e o alcoolismo crônico as mais reconhecidas. Outras causas de hipercortisolismo laboratorial são infecção bacteriana grave, obesidade principalmente visceral, síndrome dos ovários policísticos, diabetes mellitus mal controlado, síndrome de abstinência alcoólica, síndrome de resistência ao glicocorticóide generalizada, anorexia nervosa e outras doenças psiquiátricas tais como síndrome do pânico, ansiedade crônica e psicoses (1-7).

\section{FISIOPATOLOGIA}

O hipercortisolismo no estado de PC não apresenta uma causa bem definida. Acredita-se que seja principalmente secundário ao aumento de secreção de $\mathrm{CRH}$ (corticotroph hormone release) (4). Algumas teorias são descritas a seguir de acordo com as principais etiologias.

\section{Obesidade}

Diversas evidências na literatura demonstram que o eixo hipotalámo-hipófise-adrenal (HHA) apresenta-se hiper-responsivo em indivíduos obesos, principalmente naqueles com distribuição central de gordura $(8,9)$.

$\mathrm{Na}$ obesidade, é descrito um aumento do clearance metabólico do cortisol que levaria a um maior estímulo do eixo HHA (10). Acredita-se que a diminuição da globulina ligadora de corticosteróide (CBG) e a alta densidade de receptores de glicocorticóides (GC) periféricos são os principais responsáveis pelo aumento do clearance metabólico do cortisol presente nos obesos (11).

Os adipócitos viscerais possuem maior densidade de receptores de GC; dessa maneira, quanto maior a quantidade de gordura visceral maior o clearance metabólico de cortisol e, portanto, maior é o estímulo do eixo HHA $(12,13)$.

O aumento do clearance metabólico geralmente é compensado pelo aumento na produção de cor- tisol, o que geralmente torna ausente o hipercortisolismo bioquímico (G-6), mas o hipercortisolismo funcional na obesidade pode contribuir para a síndrome metabólica com características muito semelhantes à SC (14).

Estudos recentes têm demonstrado uma desregulação da atividade da enzima 11 $\beta$-hidroxiesteróide-desidrogenase do tipo 1 caracterizada por um aumento dessa atividade no tecido adiposo visceral de ratos Zuker obesos e em humanos $(15,16)$ e uma diminuição no fígado. Essa enzima catalisa a conversão de cortisona em cortisol acarretando uma maior síntese de GC local, o que está associado à hiperfagia, obesidade visceral e síndrome metabólica (15-19).

Os GC têm sido descritos como promotores da diferenciação e proliferação dos adipócitos em humanos (20).

\section{Síndrome dos Ovários Policisticos (SOP)}

A anovulaçào fisiológica da adolescência pode estar mascarada pela SOP, que sabe-se em cerca de $70 \%$ dos casos é poligênica. Quadros de obesidade severa e resistência insulínica considerados estados de PC são fatores de risco para desenvolvimento de SOP (3). Os casos de obesidade severa e de difícil tratamento são exemplos de PC na adolescência e considerados preditores de SOP.

\section{Alcoolismo}

Vários fatores influenciam o desenvolvimento do estado de PC no alcoolismo. A duração do alcoolismo e o período de abstinência podem alterar a atividade do eixo HHA. A parada abrupta na ingestão de álcool estimula o eixo HHA (talvez secundário ao estresse) e este efeito tende a diminuir com o progredir do período de abstinência (21-23). Estudos mostram que muitas vezes é necessário um período em torno de 2 a 4 meses de abstinência para que o eixo HHA volte ao estado normal (24-26). Alguns autores postulam que o estado de PC no alcoolismo representaria um hipercortisolismo induzido pelo estresse repetido aos múltiplos episódios de abstinência subaguda do álcool (27).

É valido ressaltar, ainda, que a depressão presente em 25 a $50 \%$ dos etilistas crônicos (24) e a desnutrição (28) também estimulam o eixo HHA.

Alguns trabalhos têm demonstrado uma maior ativação do eixo HHA após a ingestão aguda de álcool $(0,8-1,75 \mathrm{~g} / \mathrm{kg})$ em indivíduos saudáveis $(29,30)$, entretanto outros trabalhos não confirmaram esses achados (31). Esses dados são difíceis de serem comparados, já que diferem em duração da observa- 
ção $(2-48$ h) e nas condições basais de avaliação (jejum ou ausência de jejum).

Cobb e cols. demonstraram um aumento de esteroidogênese após adicionar etanol $(0,25-3,0 \mathrm{~g} / \mathrm{L})$ em tecidos adrenais de ratos na ausência de ACTH (adrenocorticotrophin). Este efeito não era observado quando o ACTH era adicionado ao meio, concluindo que haveria um provável efeito direto do álcool sobre as adrenais estimulando a esteroidogênese (32). Em trabalho de Elias e cols. (33) utilizando o mesmo método em adrenais humanas não se observou os mesmos resultados.

Além da hipótese adrenal, diversos autores têm sugerido uma redução parcial na resposta hipofisária tanto à estimulação pelo $\mathrm{CRH}$ quanto à depressão pelos GC causada por uma alteração na tradução do sinal mediada pelo álcool associada ao down regulation de receptores de CRH na hipófise $(34,35)$. Essa hipótese explicaria o aumento dos níveis de $\mathrm{CRH}$ liquórico em etilistas com ausência de hipercortisolismo laboratorial (35).

Um trabalho bastante interessante analisou as características histológicas da hipófise e da adrenal de ratos Wistar submetidos à ingestão alcoólica por três meses. Nesse estudo, foi encontrado um aumento na concentração de grânulos secretórios de ACTH e um espessamento da zona fasciculada e reticular nas adrenais desses ratos, demonstrando uma maior ativação do eixo HHA (36).

Frente ao fato do metabolismo do cortisol ser predominantemente hepático, diversos autores postulam que a disfunção hepática seria a principal responsável pelo hipercortisolismo induzido pelo alcoolismo crônico (teoria hepática).

Bruna e cols. demonstraram uma correlação positiva entre os níveis de cortisol sérico e o declínio da função hepática em 31 etilistas (cirróticos e não cirróticos) (37). Entretanto, Mac Donald e cols. (38) encontraram uma correlação inversa entre o score ChildPugh e os níveis de cortisol basal e após a administração de $\mathrm{ACTH}$ ou insulina em 38 cirróticos não associados ao etilismo. A comparação entre os estudos é difícil pela heterogeneidade entre os grupos, mas parece bem estabelecido que apenas distúrbios graves de função hepática podem interferir no metabolismo do cortisol ou na produção da CBG, ambos levando ao aumento dos níveis de cortisol livre $(39,40)$.

$\mathrm{O}$ fato de apenas alguns etilistas desenvolverem o estado de PC, seja clínico e/ou bioquímico, demonstra que a predisposição genética ou a sensibilidade tanto ao álcool quanto ao cortisol também influenciam na etiopatologia dessa doença (21).

\section{DEPRESSÃO MAIOR}

A literatura psiquiátrica sugere que cerca de $80 \%$ dos pacientes portadores de depressão maior apresentam hipercortisolismo (41).

Evidências demonstram uma hiperatividade do eixo HHA em indivíduos deprimidos (42) e retorno ao estado normal após remissão da depressão (43). Estudos post-mortem revelaram um aumento do número de células do núcleo para-ventricular expressando CRH ou co-expressando CRH e AVP (arginina vasopressina) $(44,45)$ em pacientes deprimidos.

Acredita-se que a hiperatividade do eixo estaria associada tanto ao aumento da liberação de AVP quanto à maior sensibilidade do eixo HHA à AVP $(46,47)$.

A resposta da ACTH ao teste do CRH está diminuída na depressão endógena e alguns trabalhos demonstram inclusive down regulation dos receptores CRHR-1, refletindo o aumento nos níveis de CRH (48).

\section{Anorexia nervosa}

Anorexia nervosa representa um modelo de hipercortisolismo funcional associado à hiper-atividade do eixo HHA assim como a maioria das causas do estado de PC. Estudos funcionais demonstram que pacientes com anorexia apresentam uma diminuição da afinidade da CBG pelo cortisol, aumentando assim os níveis de cortisol livre (49).

Semelhante ao que ocorre nas demais causas de PC, na anorexia nervosa é descrita uma menor resposta do ACTH ao teste do CRH e perda da retro-regulação do eixo HHA pelo hipercortisolismo (50,5l). Alguns autores sugerem que o aumento nos níveis de cortisol sérico estaria relacionado a uma diminuição no seu metabolismo (52).

Em trabalho recente, demonstrou-se uma redução em $138 \%$ da resposta do cortisol à AVP exógena, sugerindo uma menor sensibilidade hipofisária a esse peptídeo (o contrário do que ocorre em indivíduos deprimidos). O ganho de peso produziu um incremento dessa resposta em $55 \%$ (53).

Os níveis de AVP endógena estão elevados nos indivíduos com anorexia nervosa (54) e a saturação dos receptores AVP VIb-R ou down regulation dos mesmos levaria à diminuição da resposta do cortisol à AVP exógena. Portanto, diferente do que ocorre na depressão, a hiper-atividade do eixo HHA parece ser mediada apenas pelo CRH (55).

Apesar de alguns autores sugerirem que o hipercortisolismo correlaciona-se inversamente com o IMC (índice de massa corpórea) e desnutrição, outros acre- 
ditam que o balanço energético negativo é mais importante quando comparado ao IMC absoluto $(56,57)$.

Kudielka e cols. demonstraram que a reposição de estrógeno pode reduzir essa hiperatividade do eixo HHA através da melhora na eficácia do feedback inibitório exercido pelos GC. Efeitos semelhantes seriam obtidos durante a realimentação desses pacientes graças ao aumento da leptina, que sabidamente eleva os níveis de estrógenos (58).

A contribuição do CRH na hiperatividade do eixo HHA está associada à persistente perda de peso característica dos pacientes com anorexia nervosa, pois sabe-se que o $\mathrm{CRH}$ causa profunda inibição do apetite e diminuição do esvaziamento gástrico $(59,60)$.

\section{Síndrome de resistência generalizada ao glicocorticóide}

Uma mutação no domínio de ligação no receptor dos GC causa uma diminuição no feedback inibitório nos níveis de $\mathrm{ACTH}$, causando elevação nos níveis de cortisol sérico.

Os efeitos clássicos de hipercortisolismo (miopatia proximal, osteoporose, fragilidade vascular) geralmente estão ausentes. Os níveis de cortisol sérico e urinário estão elevados e não há supressão dos níveis séricos de cortisol sob dexametasona $1 \mathrm{mg}$ overnight. Entretanto, o quadro clínico de hiperandrogenismo pode estar presente, levando ao aparecimento de acne, hirsutismo e irregularidade menstrual e hipertensão arterial sistêmica associada ou não à hipocalemia pela ação mineralocorticóide ou saturação da $11 \beta$-hidroxiesteróide-desidrogenase pelos níveis elevados de cortisol (61). Essas duas manifestações podem trazer alguma dificuldade diagnóstica, pois também estão presentes na SC clássica. História familiar positiva, além da manutenção do ritmo circadiano, muitas vezes em nível mais elevado de cortisol sérico, pode auxiliar na diferenciação entre essas duas entidades $(1,62,63)$.

\section{DIAGNÓSTICO}

\section{Clínico}

O diagnóstico clínico do estado de PC implica na diferenciação com a SC propriamente dita. Muitas vezes, é necessário um período de observação prolongado dos pacientes portadores de queixas sugestivas, já que no PC não ocorre evolução do quadro clínico. Além disso, a identificação das possíveis causas do PC e a melhora do quadro clínico após a resolução dessas causas possibilita esse diagnóstico diferencial.
É válido ressaltar que diversas patologias estão associadas ao hipercortisolismo laboratorial, porém o estado de PC é definido apenas quando esse hipercortisolismo é acompanhado do fenótipo de SC (exceto na anorexia nervosa e depressão).

\section{Laboratorial}

Os resultados dos testes laboratoriais apresentam um overlap importante entre os indivíduos portadores de PC e SC e ainda costumam apresentar resultados diferentes de acordo com a etiologia dessa doença.

\section{Cortisol sérico à meia-noite}

É uma maneira de se avaliar o ritmo circadiano de cortisol que é caracterizado por picos entre 6-8 h e nadir entre 23-24 h.

Estudos prévios demonstram que pacientes obesos mantém o ritmo circadiano do cortisol apesar de níveis mais elevados em relação à população normal (64). Esse fenômeno também é observado em outras causas de PC (65).

Em estudo realizado por Papanicolaou e cols., foram avaliados 240 pacientes com SC (198 portadores de doença de Cushing, 27 com síndrome de ACTH ectópico e 15 com Cushing de origem adrenal) e 23 portadores de PC de diversas etiologias (depressão, anorexia, alcoolismo, diabetes mellitus mal controlado). Considerando como perda do ritmo circadiano de cortisol, níveis de cortisol sérico à meia-noite $>7,5 \mu \mathrm{g} / \mathrm{dL}$, esse teste apresentou $100 \%$ de especificidade e $96 \%$ de sensibilidade (4).

Em um estudo prévio, Newell-Price e cols. analisaram 150 portadores de SC e concluíram que um cortisol sérico à meia noite (no sono) acima de $1,8-2,0 \mu \mathrm{g} / 24 \mathrm{~h}$ distinguia esses pacientes de controles normais (66).

Quando Papanicolaou utilizou a linha de corte de 2,0 $\mu \mathrm{g} / \mathrm{dL}$ anteriormente reportada por NewellPrice, observou-se uma sensibilidade de 99\% em detrimento de uma especificidade de apenas $26 \%$, confirmando o dado de que grande parte dos portadores de PC apresentam ritmo circadiano de secreção de cortisol em vigência de hipercortisolemia (67).

\section{Cortisol salivar ( $F$ sal)}

Medidas do cortisol na saliva apresentam diversas vantagens, dentre elas: representa o cortisol livre (biologicamente ativo), é estável à temperatura ambiente, não requer a internação do paciente e é simples de ser coletada $(68,69)$.

Em estudo recente, foram avaliadas 290 mulheres (63 normais, 47 com anorexia nervosa sem tratamento 
farmacológico, $30 \mathrm{com}$ anorexia nervosa em tratamento farmacológico e 150 com obesidade central definida por circunferência abdominal/ circunferência do quadril > $0,85)$. No protocolo do estudo foram realizadas medidas do cortisol sérico e salivar às $8 \mathrm{~h}, 17 \mathrm{~h}$ e $24 \mathrm{~h}, 3$ medidas de medidas de cortisol na urina de $24 \mathrm{~h}$ e cortisol sérico e salivar após $1 \mathrm{mg}$ de dexametasona overnight (somente as anoréticas). Os resultados revelaram que as portadoras de anorexia nervosa sem tratamento apresentavam níveis de cortisol elevados em todas as medidas durante as 24 h, $14 \%$ suprimiam o cortisol sérico após $1 \mathrm{mg}$ de dexametasona, 19\% suprimiam o cortisol salivar, e que a relação do $\mathrm{F}$ sal às $8 \mathrm{~h} / 24 \mathrm{~h}$ era diminuída. Essas alterações eram menos pronunciadas naquelas que recebiam tratamento (53\% suprimiam em cortisol sérico e $35 \%$ em cortisol salivar). Nas pacientes obesas não houve correlação entre os níveis de cortisol sérico e salivar, e o IMC e os pacientes apresentava uma menor relação entre $\mathrm{F}$ sal $8 \mathrm{~h} / 24 \mathrm{~h}(79)$.

\section{Cortisol urinário de 24 h (Fu 24 h)}

A dosagem de cortisol urinário livre é considerada como um dos testes de screening de escolha para o diagnóstico de hipercortisolismo (71-73), embora se observe superposição importante de valores entre os indivíduos com SC e PC (74). No entanto, valores de $\mathrm{Fu}$ acima de $300 \mu \mathrm{g} / 24 \mathrm{~h}$ geralmente estão presentes apenas na SC.

Vários métodos estão disponíveis para a medida do cortisol urinário de $24 \mathrm{~h}$, dentre eles: radio imunoensaios e a cromatografia líquida de alta performance (HPLC).

Devido à maior especificidade do método HPLC, este vem se tornando o método de escolha para a dosagem de cortisol urinário (72), salientandose a possibilidade de fatores interferentes. Foram descritos alguns casos de pseudo-hipercortisolúria em pacientes que utilizavam fenofibrato e carbamazepina com a utilização desse método (71,73).

Num estudo realizado por Duellos e cols., que avaliou 19 mulheres portadoras de anorexia nervosa, apenas 3 apresentavam níveis elevados de Fu 24 h (75).

Em outra série de pacientes com 33 portadores de PC de diversas etiologias, 199 obesos, 91 com doença de Cushing e 27 normais, foi aplicada uma curva ROC (receive operator curve) que avaliou a performance do $\mathrm{Fu} 24 \mathrm{~h}$ em realizar o diagnóstico diferencial entre essas entidades. Considerando níveis de cortisol urinário livre acima de $80 \mu \mathrm{g}$ e $120 \mu \mathrm{g} / 24 \mathrm{~h}$, foram obtidas acurácias de $87 \%$ e $95 \%$, respectivamente.
Em estudo realizado pelo grupo de obesidade do Hospital das Clínicas de São Paulo, 9,7\% dos pacientes obesos apresentavam níveis elevados de cortisol urinário (76), enquanto que Beauregard e cols. (77) descreveram os níveis de cortisol urinário livre normais ou discretamente aumentados em pacientes obesos e raramente maiores do que quatro vezes o valor de referência.

\section{Variações circadiana e pulsátil de ACTH e cortisol}

O ACTH e o cortisol são secretados de maneira pulsátil, obedecendo ao ritmo circadiano já anteriormente descrito. Muitos estudos têm demonstrado que na DC existe uma alteração da pulsatilidade e da ritmicidade na secreção desses hormônios. Acredita-se que no PC essas alterações estejam presentes em menor intensidade (78). Analisando 31 pacientes com DC, 11 portadores de PC e 17 indivíduos controle, Cunningham e cols. demonstraram que um coeficiente de variação entre as amplitudes de secreção de ACTH ou cortisol abaixo de $40 \%$ pode discriminar corretamente esses indivíduos com $100 \%$ de sensibilidade e especificidade para os valores de cortisol e $97 \%$ de sensibilidade e $100 \%$ de especificidade para os valores de ACTH (79).

\section{Teste da desmopressina (DDAVP)}

Este teste consiste na injeção endovenosa de $10 \mu \mathrm{g}$ de DDAVP, análogo da vasopressina de longa duração, para dosagem de cortisol sérico e ACTH plasmático.

Tsagarakis e cols. (80) demonstraram que, utilizando como critério de resposta ao teste da desmopressina um aumento em $20 \%$ de cortisol sérico, $21 / 25$ pacientes com doença de Cushing apresentam testes positivos, enquanto que apenas $3 / 20$ portadores de obesidade apresentaram essa resposta (84\% sensibilidade e $85 \%$ especificidade). Considerando como critério de resposta um aumento de 50\% nos níveis de ACTH, 23/25 pacientes com doença de Cushing apresentam resposta, enquanto que $3 / 20$ obesos apresentaram teste positivo (sensibilidade de 92\% e especificidade de 85\%). Nenhum paciente portador de outras etiologias de SC apresentou respostas a esse teste. Portanto, o teste da desmopressina apresenta uma baixa sensibilidade (63\% para cortisol e $69 \%$ para ACTH) no diagnostico diferencial entre SC e obesidade.

Outro trabalho (3) avaliou 173 pacientes (76 com doença de Cushing, 30 com PC secundário à SOP, etilismo ou depressão maior, 36 obesos e 31 indivíduos normais). Após o teste do DDAVP, houve aumento importante do ACTH e cortisol nos 
pacientes com doença de Cushing, mas não naqueles com PC. Um aumento modesto, mas significante, nos níveis de ACTH também foi observado nos obesos e normais.

Recentemente, Coiro e cols. avaliaram 8 etilistas crônicos e não encontraram alteração nos níveis e ACTH e cortisol séricos após a administração de desmopressina nestes pacientes (81).

Ao utilizar como critério de resposta um aumento absoluto nos níveis de ACTH maior ou igual $6.0 \mathrm{pmol} / \mathrm{L}$ após a desmopressina (obtido através de uma curva ROC), esse teste apresentou sensibilidade de $86,8 \%$ e especificidade de $90,7 \%$ em identificar os portadores de doença de Cushing. Com a utilização de critérios clássicos $(82,83)$ para o teste do $\mathrm{CRH}$ (ACTH $\geq 35 \%$ nos tempos $15-30$ minutos e cortisol $\geq 20 \%$ nos tempos $30-45$ minutos), 71 indivíduos seriam classificados incorretamente. Além disso, observou-se uma correlação inversa entre os níveis de cortisol basal e o incremento de ACTH e cortisol após DDAVP nos indivíduos com PC, obesos e normais, reforçando a idéia de que o hipercortisolismo em condições fisiológicas ou mesmo no PC atenua a resposta ao DDAVP (84).

\section{Teste de supressão com dexametasona}

Assim como os demais testes descritos, esse teste também apresenta uma superposição importante de resultados entre os portadores de PC, SC e até indivíduos saudáveis.

O teste overnight consiste na administração de dexametasona $0,5-2,0 \mathrm{mg}$ (geralmente $1,0 \mathrm{mg}$ ) às $23-24 \mathrm{~h}$ com dosagem de cortisol sérico entre às 8-9 $\mathrm{h}$ da manhã seguinte. $\mathrm{O}$ uso de drogas que alteram o metabolismo e a absorção da dexametasona (fenitoína, carbamazepina, rifampicina) assim como estrogênios (aumentam a CBG) pode interferir nos resultados. De acordo com a literatura, esse teste apresenta $2 \%$ de resultados falso-negativos e até $30 \%$ dos indivíduos saudáveis podem apresentar resultados compatíveis com SC (85).

Em estudo retrospectivo, $18 \%$ dos pacientes portadores de doença de Cushing apresentaram níveis de Fs $<5 \mu \mathrm{g} / \mathrm{dL}$ e $8 \%$ desses apresentaram supressão para níveis abaixo de $2,0 \mu \mathrm{g} / \mathrm{dL}$ após $\mathrm{l} \mathrm{mg}$ de dexametasona overnight (86).

Conforme descrito anteriormente, a grande maioria dos portadores de PC apresenta uma hiperatividade do eixo HHA e resistência à supressão pela dexametasona. Dessa forma, é descrito que o teste da dexametasona overnight apresenta resultados falso- positivos em 23\% dos indivíduos cronicamente doentes, $13 \%$ dos obesos (87), $43 \%$ dos portadores de depressão maior e até $41 \%$ daqueles que apresentaram outras doenças psiquiátricas (88).

Recentemente, Coiro e cols. demonstraram que a dexametasona $1 \mathrm{mg}$ overnight foi incapaz de suprimir o cortisol para níveis abaixo de $5 \mu \mathrm{g} / \mathrm{dL}$ em 6 de 8 pacientes etilistas crônicos (81).

O teste de supressão com dexametasona também pode ser realizado com doses de $0,5 \mathrm{mg} 6 / 6 \mathrm{~h}$ por $48 \mathrm{~h}$. Similarmente ao teste $1 \mathrm{mg}$ overnight, este apresenta uma acurácia diagnóstica de apenas 71\% (89).

Em estudo avaliando 19 mulheres portadoras de anorexia nervosa, 7 apresentaram níveis de cortisol sérico acima de $50 \mathrm{nmol} / \mathrm{L}(1,8 \mu \mathrm{g} / \mathrm{dL})$ após dexametasona $0,5 \mathrm{mg} 6 / 6 \mathrm{~h}$ durante 48 horas (75).

\section{Teste CRH - dexametasona}

Descrito em 1993 por Yanovski e cols., este teste foi considerado o padrão ouro no diagnóstico diferencial entre SC e os estados de PC. Consiste na administração de CRH ovino $\mathrm{l} \mu \mathrm{g} / \mathrm{kg}(100 \mu \mathrm{g})$, intravenoso, $2 \mathrm{~h}$ após a última dose de dexametasona 0,5 mg 6/6h por 48 horas. Valores de cortisol sérico acima de $38 \mathrm{nmol} / \mathrm{L}(1,4 \mu \mathrm{g} / \mathrm{dL})$ obtidos no tempo 15 minutos foram observados em $100 \%$ dos indivíduos com doença de Cushing e em nenhum portador de PC (sensibilidade, especificidade e acurácia de 100\%) (74).

Em 1999, Duelos e cols. (75) questionaram a validade deste teste em pacientes portadores de anorexia nervosa, já que os mesmos não haviam sido incluídos no grupo de PC avaliado por Yanovski (74). Em seu estudo foram avaliadas 19 mulheres portadoras de anorexia nervosa pareadas com um grupo controle. Em 7 das 19 pacientes os níveis basais de cortisol sérico estavam acima de $50 \mathrm{nmol} / \mathrm{L}$, resultado não observado em nenhum dos controles. Após a administração de oCRH, 9 das 19 pacientes anoréticas exibiram valores de cortisol sérico acima da linha de corte de $38 \mathrm{nmol} / \mathrm{L}(1,4 \mu \mathrm{g} / \mathrm{dL})$ definida anteriormente por Yanovski.

Estes dados reforçam mais uma vez a superposição de valores nos exames laboratoriais entre os portadores de PC e ainda as discrepâncias fisiopatológicas entre as diversas etiologias dessa doença.

\section{Teste LVP (lisina-vasopressina) - dexametasona} $\mathrm{O}$ teste LVP - dexametasona consiste na injeção intravenosa de LVP 10 UI cedo após $\mathrm{l} \mathrm{mg}$ de dexametasona overnight. Contreras e cols. avaliaram a aplicação deste teste em 34 pacientes portadores de Síndrome de Cushing, 18 controles normais, 4 porta- 
dores de depressão maior e 5 indivíduos com aparência "cushingóide" de diversas etiologias. Nesse estudo, encontraram uma sensibilidade de $88,9 \%$ e especificidade de $100 \%$ para esse teste (89).

\section{Teste de tolerância à insulina (ITT)}

Este teste consiste na aplicação de $0,1 \mathrm{U} / \mathrm{kg}$ de insulina regular endovenosa com posteriores dosagens de glicemia, ACTH e cortisol.

Apesar da superposição de respostas entre os portadores de PC, SC e mesmo indivíduos normais, este teste tem sido utilizado para esse diagnóstico diferencial. Os pacientes deprimidos geralmente apresentam uma resposta do cortisol intacta à hipoglicemia, enquanto que apenas 18\% de portadores de SC mantêm essa resposta.

É válido ressaltar que pela resistência insulínica associada ao hipercortisolismo muitas vezes torna-se necessário utilizar doses de até $0,3 \mathrm{U} / \mathrm{kg}$ de insulina regular para que ocorra hipoglicemia nesses pacientes.

\section{Teste de loperamida}

A loperamida é um agonista opióide que tem sido utilizado no diagnóstico diferencial entre SC e os estados de PC.

Esse teste consiste na administração oral de loperamida $16 \mathrm{mg}$ às $8 \mathrm{~h}$ com dosagem de cortisol sérico 3,5 h após. A loperamida causa inibição do $\mathrm{CRH}$ (90) e, assim, supressão dos níveis de ACTH e cortisol em normais, o que não é observado em portadores de SC $(91,92)$.

Após avaliar os dados em conjunto dos diversos relatos de uso deste teste, foram avaliados 49 portadores de SC (42 com doença de Cushing, 2 com síndrome de secreção ectópica de ACTH e 5 com tumores adrenais) que não apresentaram supressão dos níveis de cortisol sérico abaixo de $5 \mu \mathrm{g} / \mathrm{dL}$ ( $138 \mathrm{nmol} / \mathrm{L}$ ) após administração de dexametasona 1 mg overnight. Em 128 de 138 indivíduos normais ou portadores de PC de diversas etiologias, observou-se supressão dos níveis de cortisol sérico abaixo deste valor, resultando em uma sensibilidade de $100 \%$ e especificidade de $93 \%$ para esse teste $(91,92)$. A análise de um maior número de pacientes e controles será necessária para uma melhor avaliação da acurácia deste teste.

Dados recentes e com um maior número de participantes são necessários para uma melhor avaliação da acurácia deste teste.

\section{Teste do naloxone}

Naloxone é um antagonista opióide que tem sido utilizado para o diagnóstico diferencial entre o estado de PC relacionado ao etilismo crônico e à SC.
Alguns autores acreditam que o álcool determina a redução dos níveis dos peptídeos opióides endógenos levando à hiperatividade do eixo HHA e, sendo o naloxone um antagonista opióide, esse efeito no eixo seria reduzido.

Em 1995, Inder e cols. avaliaram os níveis de cortisol, ACTH, CRH e AVP a cada 20 minutos durante 2 horas em 9 etilistas e os compararam a 9 indivíduos controle pareados para sexo e idade (93). Após essa avaliação inicial eram administrados $20 \mathrm{mg}$ de naloxone intravenosos com posterior dosagem dos mesmos hormônios nos tempos $15,30,45,60,90$ e 120 minutos. O nível basal dos diversos hormônios não variou entre os dois grupos, porém os etilistas apresentaram um menor incremento dos níveis de ACTH após o naloxone (93).

\section{Teste DDAVP - dexametasona}

Devido ao fato de o DDAVP ser droga disponível e de baixo custo em nosso meio e a não disponibilidade e alto custo do CRH no Brasil (94), Salgado e cols. avaliaram a utilização do teste do DDAVP $10 \mu \mathrm{g}$ intravenosos, após dexametasona $2 \mathrm{mg}$ overnight em indivíduos normais e pacientes com doença de Cushing. Valores absolutos de cortisol sérico $\geq 2,4 \mu \mathrm{g} / \mathrm{dL}$ no tempo 15 minutos do teste puderam classificar corretamente esses indivíduos.

\section{Cateterismo bilateral e simultâneo dos seios petrosos inferiores (BIPSS)}

O BIPSS é hoje considerado o método padrão ouro para o diagnóstico da etiologia da SC ACTH-dependente, atingindo uma acurácia de quase 100\% (95).

Acredita-se que o hipercortisolismo dos estados de PC é resultado do aumento na secreção de $\mathrm{CRH}$ agindo sob o eixo hipófise-adrenal. Em contrapartida, a secreção de $\mathrm{CRH}$ pelo hipotálamo está suprimida pelo hipercortisolismo da SC. Levando em consideração esse raciocínio, Yanovsky e cols. postularam que os níveis de CRH avaliados durante o BIPSS permitiriam diferenciar essas duas situações (96).

Estudando 25 pacientes com doença de Cushing, 17 com síndrome de secreção ectópica de ACTH, 7 com SC de origem adrenal, 6 portadores de PC e 11 voluntários normais, Yanovsky e cols. não encontraram diferenças significativas nos níveis de $\mathrm{CRH}$ nos seios petrosos (esfenoidal) ou em veia periférica nos diversos grupos. Todos os indivíduos apresentaram níveis de $\mathrm{CRH}$ indetectáveis e apenas os valores de ACTH eram mais elevados nos portadores de doença de Cushing (96). Portanto, o BIPSS não parece acrescentar no diagnóstico diferencial entre a SC e os estados de PC. 


\section{Teste da hexarelina}

A hexarelina é um peptídeo que estimula a secreção de ACTH hipofisário de uma maneira diferente da desmopressina (97). Acredita-se que a hexarelina age diretamente no hipotálamo, aumentando o ACTH via $\mathrm{CRH}$ endógeno ou outros fatores liberadores de ACTH hipotolâmicos.

Evidências mostram que após a desconexão hipotálamo-hipofisária, o efeito da hexarelina é completamente abolido $(97,98)$ e que ainda essa droga é capaz de estimular a secreção de ACTH in vitro $(99,100)$.

O teste com hexarelina consiste na administração de $2,0 \mu \mathrm{g} \cdot \mathrm{kg}^{-1}$ com dosagem de ACTH e cortisol nos mesmos tempos do teste da desmopressina.

Coiro e cols. avaliaram os resultados do teste da hexarelina em 8 etilistas com PC, 6 portadores de doença de Cushing e 9 controles normais. Como já era de se esperar, a hexarelina levou a uma reposta exagerada de ACTH e cortisol nos portadores de doença de Cushing e um aumento menos intenso nos indivíduos normais, não se observando resposta nos etilistas (81). Conclui-se que este teste seria de grande valor no diagnóstico diferencial entre os portadores de PC de etiologia alcoólica. Mais estudos são necessários para avaliar sua utilização nas demais causas do estado de PC.

\section{Antígeno prostático sérico específico (PSA)}

Recentemente, a dosagem do PSA através de um imunoensaio enzimático por quimioiluminescência altamente sensível possibilitou a detecção do PSA no soro de mulheres. Embora o sítio exato de secreção do PSA ainda seja desconhecido nas mulheres, acredita-se que vários tecidos secretam esse hormônio via estimulação de hormônios esteróides (101).

O PSA é considerado um marcador de atividade androgênica nas mulheres (102-104) e recentemente estudos in vitro demonstraram que a sua produção em células tumorais da mama é mediada por receptores androgênicos mas também por receptores de GC e de progesterona $(105,106)$.

Estudo recente avaliou os níveis de PSA em 10 mulheres portadoras de PC dependente do álcool, 8 mulheres com doença de Cushing e 15 mulheres controles. Foram demonstrados níveis de PSA séricos significantemente maiores nas portadoras de doença de Cushing em relação às outras etiologias, e os níveis de PSA entre as etilistas e as normais foram semelhantes (106). Em conclusão, a avaliação dos níveis séricos de PSA total através de um ensaio de alta sensibilidade representa uma ferramenta a mais no diagnóstico diferencial entre essas duas entidades.

\section{TRATAMENTO}

O tratamento dos estados de PC consiste no tratamento específico das diversas etiologias relacionadas a ele. A melhora das queixas clínicas e laboratoriais sugestivas de hipercortisolismo à medida que ocorre melhora da doença primária causadora reforçaria ainda mais o diagnóstico de PC.

\section{CONCLUSÃo}

Pseudo-Cushing é um estado clínico caracterizado pela presença do fenótipo clínico similar à síndrome de Cushing associado a algum grau de hipercortisolismo endógeno.

Hipercortisolismo laboratorial pode estar presente em diversas doenças, no entanto o estado de pseudo-Cushing está bastante estabelecido no alcoolismo crônico e na depressão maior.

O diagnostico diferencial com a síndrome de Cushing muitas vezes é bastante difícil, tornando necessária a realização de múltiplos testes laboratoriais e dinâmicos, já que nenhum pode ser considerado ideal.

Muitas vezes a observação em longo prazo permite essa diferenciação, já que o pseudo-Cushing não é doença progressiva e tende a regredir quando a sua etiologia é revertida.

\section{REFERÊNCIAS}

1. Newell-Price J, Trainer P, Besser M, Grossman A. The diagnosis and differential diagnosis of Cushing's syndrome and pseudo-Cushing's states. Endocr Rev 1998; 19(5):647-72.

2. Pecori Giraldi F, Pivonello R, Ambrogio AG, De Martino MC, De Martin M, Scacchi M, et al. The dexamethasone-suppressed corticotropin-releasing hormone stimulation test and the desmopressin test to distinguish Cushing's syndrome from pseudo-Cushing's states. Clin Endocrinol 2007; 66(2):251-7

3. Moro M, Putignano P, Losa M, Invitti C, Maraschini C, Cavagnini $F$. The desmopressin test in the differential diagnosis between Cushing's disease and pseudo-Cushing States. J Clin Endocrinol Metab 2000;85(10):3569-74.

4. Papanicolaou D, Yanovski J, Cutler G, Chrousos G, Nieman L. A single midnight serum cortisol measurement distinguishes Cushing's syndrome from pseudo-Cushing states. J Clin Endocrinol Metabolism 1998;83(4):1163-7.

5. Lamberts SW, Klijn JG, de Jong FH, Birkenhager JC. Hormone secretion in alcohol-induced pseudo-Cushing's syndrome. Differential diagnosis with Cushing disease. JAMA 1979;242:1640 -3.

6. Wand GS, Dobs AS. Alterations in the hypothalamic-pituitary-adrenal axis in actively drinking alcoholics. J Clin Endocrinol Metab 1991;72:1290-5.

7. Roy MS, Roy A, Gallucci WT, Collier B, Young K, Kamilaris TC, et al. The ovine corticotropin-releasing hormone-stimulation test in type I diabetic patients and controls: suggestion of mild chronic hypercortisolism. Metabolism 1993; 42:696-700. 
8. Duclos M, Gatta B, Corcuff JB, Rashedi M, Pehourcq F, Roger $P$. Fat distribution in obese women is associated with subtle alterations of the hypothalamic-pituitary-adrenal axis activity and sensitivity to glucocorticoids Clin Endocrinol 2001; 55:447-54

9. Lordelo RA, Mancini MC, Cercato C, Halpern A. Eixos hormonais na obesidade: Causa ou efeito? Arq Bras Endocrinol Metab 2007;51:34-41.

10. Salehi M, Ferenczi A, Zumoff B. Obesity and cortisol status. Horm Metabol Res 2005;37(4):193-7.

11. Rebuffe-Scrive M, Bronnegard M, Nilsso A, Eldh J, Gustafsson JA, Bjorntorp $P$. Steroid hormone receptors in human adipose tissues. J Clin Endocrinol Metab 1990;71:1215-9.

12. Bronnegard M, Arner P, Hellstrom Let, Akner G, Gustafsson JA. Glucocorticoid receptor messenger ribonucleic acid in different regions of human adipose tissue. Endocrinology 1990;127:1689-96.

13. Lottenberg SA, Giannella-Neto D, Derendorf $H$, Rocha M, Bosco A, Carvalho SV, et al. Effect of fat distribution on the pharmacokinetics of cortisol in obesity. Int J Clin Pharmacol Ther 1998;36(9):501-5.

14. Bujalska IJ, Kumar S, Stewart PM. Does central obesity reflect 'Cushing's disease of the omentum'? Lancet 1997; 349:1210-3.

15. Livingstone DE, Kenyon CJ, Walker BR. Mechanisms of dysregulation of 11 beta-hydroxysteroid dehydrogenase type 1 in obese Zucker rats. J Endocrinol 2000;167(3):533-9.

16. Masuzaki H, Paterson J, Shinyama H, Morton NM, Mullins JJ, Seckl JR, et al. A transgenic model of visceral obesity and the metabolic syndrome. Science 2001;294(5549):2166-70.

17. Rask $E$, Olsson $T$, Soderberg $S$, Andrew R, Livingstone DE, Johnson $\mathrm{O}$, et al. Tissue-specific dysregulation of cortisol metabolism in human obesity. J Clin Endocrinol Metab 2001;86(3):1418-21.

18. Katz JR, Mohamed-Ali V, Wood PJ, Yudkin JS, Coppack SW. An in vivo study of the cortisol-cortisone shuttle in subcutaneous abdominal adipose tissue. Clin Endocrinol 1999; 50:63-8.

19. Engeli S, Bohnke J, Feldpausch M, Gorzelniak K, Heintze U, Janke J, et al. Regulation of 11 beta-HSD genes in human adipose tissue: influence of central obesity and weight loss. Obes Res 2004:12(1):9-17.

20. Hauner H, Entenmann G, Wabitsch M, Gaillard D, Ailhaud G, Negrel R, et al. Promoting effect of glucocorticoids on the differentiation of human adipocyte precursor cells cultured in a chemically defined medium. J Clin Invest 1989; 84:1663-70.

21. Veldman RG, Meinders AE. On the mechanism of alcoholinduced pseudo-Cushing's syndrome. Endoc Rev 1996; 17(3):268.

22. Wilkins JN, Gorelick DA, Nademanee K, Taylor A, Herzberg DS. Hypothalamic-pituitary function during alcohol exposure and withdrawal and cocaine exposure. Recent Dev Alcohol 1992; $10: 57-71$

23. Noth $\mathrm{RH}$, Walter RM. The effects of alcohol on the endocrine system. Med Clin North Am 1984;68:133-46.

24. Dackis C, Stuckey R, Gold M, Pottash A. Dexamethasone suppression testing of depressed alcoholics. Alcohol Clin Exp Res 1986; 10:59-60.

25. Lamberts SWJ, Klijn JGM, de Jong FH, Birkenhlger JC. Hormone secretion in alcohol-induced pseudo-Cushing's syndrome: differential diagnosis with Cushing's disease. JAMA 1979;242:1640-3.

26. Jenkins RM, Page MM. Atypical case of alcohol-induced Cushingoid syndrome. Br Med J 1981;282:1117-8.

27. Elias AN, Meshkinpour H, Valenta LJ, Grossman MK. PseudoCushing's syndrome: the role of alcohol. J Clin Gastroenterol 1982;4(2):137-9.

28. Bode C, Martini G, Bode J. Effect of alcohol on microsomal cortisol and 5-alpha reductase in the liver of rats that are on a standard or low protein diet. Horm Metab Res 1978; 10:63-4.
29. Bellet S, Roman L, DeCastro O, Herrera M. Effect of acute ethanol intake on plasma II-hydroxycorticosteroid levels. Metabolism 1970;19:664-7.

30. Ylikahri RH, Huttunen $\mathrm{MO}$, Harkonen $\mathrm{M}$, Leino $\mathrm{T}$, Helenius $\mathrm{T}$, Liewendahl K, et al. Acute effects of alcohol on the anterior pituitary secretion of the tropic hormones. J Clin Endocrinol Metab 1978;46:715-20.

31. Ida Y, Tsujimaru S, Nakamaura K, Shirao I, Mukasa H, Egami $H$, et al. Effects of acute and repeated alcohol ingestion on hypothalamic-pituitary-gonadal and hypothalamic-pituitaryadrenal functioning in normal males. Drug Alcohol Depend 1992;31:57-64.

32. Cobb CF, Van Thiel DH, Gavaler JS, Lester R. Effects of ethanol and acetaldehyde on the rat adrenal. Metabolism 1981;30:537-43.

33. Elias AN, Meshkinpour H, Valenta LJ, Grossman MK. PseudoCushing's syndrome: the role of alcohol. J Clin Gastroenterol 1982;4:137-9.

34. Dave JR, Eiden LE, Karanian JW, Eskay RL. Ethanol exposure decreases pituitary corticotropin-releasing factor binding, adenylate cyclase activity, proopiomelanocortin biosynthesis and plasma kendorphin levels in the rat. Endocrinology 1986; $118: 280-6$

35. Rivier C, Bruhn T, Vale W. Effect of ethanol on the hypothalamic-pituitary-adrenal axis in the rat: role of corticotropinreleasing factor (CRF). J Pharmacol Exp Ther 1984; 229:127-31.

36. Somer L, Matavulj M, Hadzi B, Vuckovi N. The hypophysealadrenal axis in chronic alcoholism. Source Medicinski Pregled 1996;49(9-10):349-55.

37. Burra P, Franklyn JA, Ramsden DB, Elias E, Sheppard MC. Severity of alcoholic liver disease and markers of thyroid and steroid status. Postgrad Med 1992;168:804-10.

38. McDonald-JA, Haidelsman DJ, Dilworth P, Conway AJ, McCaughan GW. Hypothalamic-pituitary adrenal function in end-stage non-alcoholic liver disease. J Gastroenterol Hepato 1993; $18: 247-53$

39. Rosman PM, Farg A, Benn R, Tito J, Mishik A, Wallace EZ. Modulation of pituitary-adrenocortical function: decreased secretory episodes and blunted circadian rhythmicity in patients with alcoholic liver disease. J Clin Endocrinol Metab 1982;55:709-17.

40. McCann VJ, Fulton TT. Cortisol metabolism in chronic liver disease. J Clin Endocrinol Metab 1975:40:1038.

41. Pfohl B, Sherman B, Schlechte J, Stone B. Pituitary-adrenal axis rhythm disturbances in psychiatric depression. Arch Gen Psychiatry 1985;42:897.

42. Amsterdam JD, Maislin G, Winokur A, Berwish N, Kling M, Gold P. The oCRH stimulation test before and after clinical recovery from depression. J Affect Disord 1988; 14:213-22.

43. Pfohl B, Sherman B, Schlechte J, Winokur G. Differences in plasma ACTH and cortisol between depressed patients and normal controls. Biol Psychiatry 1985; 20:1055.

44. Purba JS, Hoogendijk WJ, Hofman MA, Swaab DF. Increased number of vasopressin- and oxytocin-expressing neurons in the paraventricular nucleus of the hypothalamus in depression. Arch Gen Psychiat 1996;53:137-43.

45. Raadsheer FC, Hoogendijk WJ, Stam FC, Tilders FJ, Swaab DF. Increased numbers of corticotropin-releasing hormone expressing neurons in the hypothalamic paraventricular nucleus of depressed patients. Neuroendocrinology 1994; 60:436-44.

46. Oshima A, Yamashita S, Owashi T, Murata T, Tadokoro C, Miyaoka $\mathrm{H}$, et al. The differential ACTH responses to combined dexamethasone/CRH administration in major depressive and dysthymic disorders. J Psychiat Res 2000; 34:325-8.

47. Von Bardeleben U, Holsboer F. Cortisol response to a combined dexamethasone/human corticotrophin-releasing hormone challenge in patients with depression. J Neuroendocrinol 1989;1:485-8. 
48. Newport DJ, Heim C, Owens MJ, Ritchie JC, Ramsey CH, Bonsall R, et al. Cerebrospinal fluid corticotropin-releasing factor (CRF) and vasopressin concentrations predict pituitary response in the CRF stimulation test: a multiple regression analysis. Neuropsychopharmacology 2003;28:569-76.

49. Casper RC, Chatterton RT, Davis JM. Alterations in serum cortisol and its binding characteristics in anorexia nervosa. $\mathbf{J}$ Clin Endocrinol Metab 1979;49:406-11.

50. Estour B, Pugea M, Lang F, Lejeune H, Broutin F, Pellet J, et al. Rapid escape of cortisol from suppression in response to IV dexamethasone in anorexia nervosa. Clin Endocrinol 1990;33:45-52.

51. Cavagnini F, Invitti C, Passamonti M, Polli EE. Response of ACTH and cortisol to corticotropin-releasing hormone in anorexia-nervosa. N Eng J Med 1986;314:184-5.

52. Boyar RM, Hellman LD, Roffwarg H, Katz J, Zumoff B, O'Connor $\mathrm{J}$, et al. Cortisol secretion and metabolism in anorexia nervosa. N Eng J Med 1977;296:190-3.

53. Connan F, Lightman SL, Landau S, Wheeler M, Treasure J, Campbell IC. An investigation of hypothalamic-pituitaryadrenal axis hyperactivity in anorexia nervosa: the role of CRH and AVP. J Psychiatr Res 2007;41(1-2):131-43.

54. Gold PW, Gwirtsman H, Avgerinos PC, Nieman LK, Gallucci WT, Kaye W, et al. Abnormal hypothalamic-pituitary-adrenal function in anorexia nervosa. Pathophysiologic mechanisms in underweight and weight-corrected patients. N Eng J Med 1986;314:1335-42.

55. Malerbi DA, Fragoso MC, Vieira Filho AH, Brenlha EM, Mendonça BB. Cortisol and adrenocorticotropin response to desmopressin in women with Cushing's disease compared with depressive illness. J Clin Endocrinol Metab 1996; 81(6):2233-7.

56. Berger M, Pirke KM, Doerr P, Krieg C, von Zersseen D. Influence of weight loss on the dexamethasone suppression test. Arch Gen Psychiat 1983;40:585-6.

57. Licinio J, Wong ML, Gold PW. The hypothalamic-pituitaryadrenal axis in anorexia nervosa. Psychiat Res 1996; 62:75-83.

58. Ahima RS, Prabakaran D, Mantzoros C, Qu D, Lowell B, Maratos FE, et al. Role of leptin in the neuroendocrine response to fasting. Nature 1996;382:250-2.

59. Glowa JR, Gold PW. Corticotropin releasing hormone produces profound anorexigenic effects in the rhesus monkey. Neuropeptides 1991; 18:55-61.

60. Arase K, York DA, Shimizu H, Shargill N, Bray GA. Effects of corticotropin-releasing factor on food intake and brown adipose tissue thermogenesis in rats. Am J Physiol 1988; 255:255-9.

61. Bronnegard M, Stierna P, Marcus C. Glucocorticoid resistant syndromes - molecular basis and clinical presentations. J Neuroendocrinol 1996;8:405-15.

62. Lamberts SW. The glucocorticoid insensitivity syndrome. Horm Res 1996;45(suppl 1):2-4.

63. Arai K, Chrousos GP. Syndromes of glucocorticoid and mineralocorticoid resistance. Steroids 1995;60:173-9.

64. Strain GW, Zumoff B, Strain JJ. Cortisol production in obesity. Metabolism 1980;29:980-5.

65. Pirich K, Vierhapper H. 24-hour serum concentration profile of cortisol in patients with Cushing's disease. Exp Clin Endocrinol 1988;92:275-9.

66. Newell-Price J, Trainer P, Perry L, Wass J, Grossman A, Besser M. A single sleeping midnight cortisol has $100 \%$ sensitivity for the diagnosis of Cushing's syndrome. Clin Endocrinol 1995; $43: 545-50$

67. Cunningham JM, Buxton OM, Weiss RE. Circadian variation in Cushing's disease and pseudo-Cushing states by analysis of $F$ and ACTH pulsatility. J Endocrinol Invest 2002; 25(9):791-9.

68. Riad-Fahmy D, Read GF, Walker RF, Grifths K. Steroids in saliva for assessing endocrine function. Endocr Rev 1982; 3:367-95.

69. Chen YM, Cintron NM, Whitson PA. Long-term storage of salivary cortisol samples at room temperature. Clin Chem 1992;38:304-5.
70. Putignano P, Dubini A, Toja P, Invitti C, Bonfanti S, Redaelli G, et al. Salivary cortisol measurement in normal-weight, obese and anorexic women: comparison with plasma cortisol. Eur J Endocrinol 2001;145:165-71.

71. Meikle AW, Findling J, Kushnir MM, Rockwood Al, Nelson GJ, Terryj AH. Pseudo-Cushing syndrome caused by fenofibrate interference with urinary cortisol assayed by high-performance liquid chromatography. Clin Endocrinol Metab 2003;88(8):3521-4.

72. Turpeinen U, Markkanen H, Valimaki M, Stenman UH. Determination of urinary free cortisol by HPLC. Clin Chem 1997; 43:1386-91.

73. Findling J, Pinkstaff S, Shaker JL, Raff H, Nelson J. Pseudohypercortisoluria: spurious elevation of urinary cortisol due to carbamazepine. Endocrinologist 1998;8:51-4.

74. Yanovski JA, Cutler Jr GB, Chrousos GP, Nieman LK. Corticotropin-releasing hormone stimulation following low-dose dexamethasone administration. A new test to distinguish Cushing's syndrome from pseudo-Cushing's states. JAMA 1993;269:2232-8.

75. Duclos M, Corcuff JB, Roger P, Tabarin. A. The dexamethasone-suppressed corticotrophin-releasing hormone stimulation test in anorexia nervosa. Clin Endocrinol 1999; 51(6):725-31.

76. Arguello AMC, Cercato $C$, Barroso RL, et al. Urinary free cortisol levels in obese patients. Int J Obes Relat Metab Disord 2002;26(suppl. 1):S198.

77. Beauregard C, Dickstein G, Lacroix A. Classic and recent etiologies of Cushing syndrome: diagnosis and therapy. Treat Endocrinol 2002;1(2):79-94.

78. Deuschle M, Schweiger U, Weber B, Gotthardt U, Korner A, Schmider J, et al. Diurnal activity and pulsatility of the hypothalamus-pituitary-adrenal system in male depressed patients and healthy controls. J Clin Endocrinol Metab 1997;82(1):234-8.

79. Cunningham JM, Buxton OM, Weiss RE. Circadian variation in Cushing's disease and pseudo-Cushing states by analysis of $F$ and ACTH pulsatility. J Endocrinol Invest 2002;25(9):791-9.

80. Tsagarakis S, Vasiliou V, Kokkoris P, Stavropoulos G, Thalassinos N. Assessment of cortisol and ACTH responses to the desmopressin test in patients with Cushing's syndrome and simple obesity. Clin Endocrinol 1999;51(4):473-7.

81. Coiro V, Volpi R, Capretti L, Caffarri G, Chiodera P. Desmopressin and hexarelin tests in alcohol-induced pseudo-Cushing's syndrome. J Intern Med 2000;247(6):667-73.

82. Newell-Price J, Perry L, Medbak S, Monson J, Savage M, Besser $\mathrm{M}$, et al. A combined test using desmopressin and corticotropin-releasing hormone in the differential diagnosis of Cushing's syndrome. J Clin Endocrinol Metab 1997; 82:176-81.

83. Niemann LK, Oldfield EH, Wesley $R$, Chrousos GP, Loriaux DL, Cutler Jr GB. A simplified morning ovine corticotropinreleasing hormone stimulation test for the differential diagnosis of adrenocorticotropin-dependent Cushing's syndrome. J Clin Endocrinol Metab 1993;77:1308-12.

84. Gold PW, Loriaux DL, Roy A, Kling MA, Calabrese JR, Kellner $\mathrm{CH}$, et al. Responses to corticotropin-releasing hormone in the hypercortisolism of depression and Cushing's disease. Pathophysiologic and diagnostic implications. N Engl J Med 1986;314:1329-35.

85. Tsigos C, Papanicolaou DA, Chrousos GP. Advances in the diagnosis and treatment of Cushing's syndrome. Bailliere Clin Endocrinol Metab 1995;9:315-36.

86. Findling JW, Raff H, Aron DC. The low-dose dexamethasone suppression test: a reevaluation in patients with Cushing's syndrome. J Clin Endocrinol Metab 2004;89:1222.

87. Crapo L. Cushing's syndrome: a review of diagnostic tests. Metabolism 1979;28:955-77.

88. Murphy BE. Steroids and depression. J Steroid Biochem Mol Biol 1991;38:537-59.

89. Contreras $\mathrm{P}$, Araya V. Overnight dexamethasone pre-treatment improves the performance of the lysine-vasopressin test in the diagnosis of Cushing's syndrome. Clin Endocrinol 1996;44:703-10. 
90. Auernhammer CJ, Stalla GK, Lange M, Pfeiffer A, Muller OA. Effects of loperamide on the human hypothalamo-pituitary-adrenal axis in vivo and in vitro. J Clin Endocrinol Metab 1992;75:552-7.

91. Ambrosi B, Bochicchio D, Ferrario R, Colombo P, Faglia G. Effects of the opiate agonist loperamide on pituitary-adrenal function in patients with suspected hypercortisolism. J Endocrinol Invest 1989;12:31-5.

92. Ambrosi B, Bochicchio D, Colombo P, Fadin C, Faglia G. Loperamide to diagnose Cushing's syndrome. JAMA 1993; 270:2301-2.

93. Inder WJ, Joyce PR, Ellis MJ, Evans MJ, Livesey JH, Donald RA. The effects of alcoholism on the hypothalamic-pituitaryadrenal axis: interaction with endogenous opioid peptides. Clin Endocrinol 1995;43(3):283-90.

94. Salgado LR, Knoepfelmacher M, Pimentel F, Wajchenberg BL, Liberman B. Desmopressin (DDAVP): a more reliable test for diagnosis of Cushing's disease. Programs and Abstracts of the Endocrine Society 80th Annual Meeting 1998; New Orleans, LA:69.

95. Oldfield EH, Nieman LK. Petrosal sinus sampling with and without corticotropin releasing hormone for the differential diagnosis of Cushing's syndrome. N Engl J Med 1991; 325:897-905.

96. Yanovski JA, Nieman LK, Doppman JL, Chrousos GP, Wilder $\mathrm{RL}$, Gold PW, et al. Plasma levels of corticotropin-releasing hormone in the inferior petrosal sinuses of healthy volunteers, patients with Cushing's syndrome, and patients with pseudo-Cushing states. J Clin Endocrinol Metab 1998; 83(5):1485-8.

97. Hickey GJ, Drisko J, Faidley T, Chang C, Anderson L, Nicolich $S$, et al. Mediation by the central nervous system is critical to the in vivo activity of the GH secretagogue L-692, 585. J Endocrinol 1996;148:371-80.

98. Wand GS, Dobs AS. Alterations in the hypothalamic-pituitary-adrenal axis in actively drinking alcoholics. J Clin Endocrinol Metab 1991;72:1290-5.

99. Bowers CY, Momany FA, Reynolds GA, Hong A. On the in vitro and in vivo activity of a new synthetic hexapeptide that acts on the pituitary to specifically release growth hormone. Endocrinology 1984;114:1537-45.
100.Arvat E, De Vito L, Macacgno B, Broglio F, Boghen MF, Deghenghi R, et al. Effects of GHRF-2 and hexarelin, two synthetic $\mathrm{GH}$-releasing peptides, on $\mathrm{GH}$, prolactin, ACTH and cortisol levels in men. Comparison with the effects of GHRH, TRH and hCRH. Peptides 1997; 18:885-91.

101.Diamandis EP, Yu H. New biological functions of prostate specific antigen? J Clin Endocrinol Metab 1995;80:1515-7.

102. Melegos DN, Yu H, Ashok M, Wang C, Stanczyk F, Diamandis EP. Prostate specific antigen in female serum. A potential new marker of androgen excess. J Clin Endocrinol Metab 1997;82:777-80.

103.Negri C, Tosi F, Dorizzi R, Fortunato A, Spiazzi GG, Muggeo $M$, et al. Antiandrogen drugs lower serum prostate-specific antigen (PSA) levels in hirsute subjects: Evidence that serum PSA is a marker of androgen action in women. J Clin Endocrinol Metab 2000;85:81-4.

104.Obiezu CV, Scorilas A, Magklara A, Thornton MH, Wang CY, Stanczyk FZ, et al. Prostate-specific antigen and human glandular kallikrein 2 are markedly elevated in urine of patients with polycystic ovary syndrome. J Clin Endocrinol Metab 2001;86:1558-61.

105.Luke MC, Coffey DS. Human androgen receptors binding to the androgen response element of prostate specific antigen. J Androl 1994;15:49-51.

106. Coiro V, Volpi R, Gallia P, Manfredi G, Magotti MG, SaccaniJotti G, et al. Serum total prostate-specific antigen assay in women with Cushing's disease or alcohol-dependent pseudo-Cushing's state. Horm Res 2004;61:148-52.

Endereço para correspondência:

Luiz Roberto Salgado

Av. Brigadeiro Luiz Antônio 4258

01402-002 São Paulo, SP

Fax: (1 1) 3885-6719

E-mail: salga@uol.com.br 